\title{
Un sentido teleológico regulativo de la nada en el pensamiento de Mainländer
}

\section{A Teleological Regulatory Sense of Nothingness in Mainländer's Thought}

\author{
PAOLO GAJARDO JAÑA \\ UNIVERSIDAD DE CHILE
}

Recibido: 25/01/2020 Aceptado:07/04/2020

\section{RESUMEN}

Dentro de la filosofía de Mainländer, la nada posee un rol fundamental. La nada posee un sentido teleológico regulativo, es decir, es el fin o meta del universo, como resultado del primer movimiento: la muerte de Dios. Para comprender cómo este autor llega a plantear dicha tesis, es necesario sondear los pasos previos que confluyen en tal conclusión. Por ello, algunos de los aspectos centrales de esta filosofía serán expuestos en este artículo, con el fin de comprender la fundamentación de esta propuesta. Y así, finalmente aclarar el rol excepcional que se le atribuye en este pensamiento a la nada.

\section{PALABRAS CLAVE \\ NADA, TELEOLOGÍA, MAINLÄNDER, DIOS, VOLUNTAD.}

\begin{abstract}
In Mainländer's philosophy, nothingness has a fundamental role. Nothingness has a teleological regulatory sense; that is to say, it is the end or goal of the universe, as a result of the first movement: the death of God. To understand how this author manages to expound that thesis, it is necessary to explore the previous steps that lead to such conclusion. Thus, some of the central aspects of this philosophy will be presented throughout this article, in order to understand the foundation of this proposal. Finally, the exceptional role that is attributed to the nothingness on this thought will be clarified.
\end{abstract}

KEYWORDS

NOTHINGNESS, TELEOLOGY, MAINLÄNDER, GOD, WILL

(C) Contrastes. Revista Internacional de Filosofía, vol. XXV №2 (2020), pp. 79-97. ISSN: 1136-4076

Departamento de Filosofía, Universidad de Málaga, Facultad de Filosofía y Letras Campus de Teatinos, E-29071 Málaga (España) 


\section{INTRODUCCIÓN}

PhILIPP BATZ MAINLÄNDER FUE UN pensador y filósofo autodidacta alemán que, pese a ser poco recordado o reconocido dentro de los grandes de la filosofía, influyó en varios pensadores que le sucedieron, tales como Nietzsche, Alfred Kubin, Jorge Luis Borges y Cioran (Volpi, 2005, p. 50), incluso, en una manera indirecta también su pensamiento repercutió en el ámbito psicoanalítico sobre Freud (Burgos Cruz \& Godoy Patiño, 2015, p. 12).

Las recientes traducciones de sus obras representan un significativo aporte para los hispanohablantes, tanto en el ámbito investigativo como divulgativo. Actualmente se encuentran traducidas sus incursiones en el ámbito literario Diario de un poeta (en la editorial Plaza y Valdés, 2015) y Rupertine del Fino (en la editorial Guillermo escolar, 2018). Asimismo, en el terreno filosófico se cuenta con la traducción del primer volumen de su obra magna la Filosofía de la redención (por la editorial Xorki, 2014), y con una antología del primer y segundo volumen (por la editorial Fondo de Cultura Económica, 2011), a la cual se ha sumado otra antología que reúne otra selección de sus textos filosóficos: Filosofía de la redención (y otros textos). Antología (publicada en la editorial Alianza el año 2020).

En su obra capital la Filosofia de la redención (Die Philosophie der Erlösung) desarrolla una cosmovisión novedosa -a través de una Analítica de la facultad cognoscitiva, una Física, Estética, Ética, Política y Metafísicaen la cual se pretende aclarar el misterio del origen del universo, y con ello, comprender el curso y destino de la humanidad.

Dentro de esta propuesta filosófica la nada -o el no-ser- posee un rol fundamental. Para Mainländer, la nada tiene un sentido teleológico regulativo, es decir, es el fin o meta del universo. En la medida en que todo se dirige hacia ella con estricta necesidad, como resultado del primer movimiento: la muerte de Dios, entendida como un suicidio divino. Para comprender cómo es que este autor llega a plantear dicha tesis, es necesario sondear los pasos previos que confluyen en tal conclusión. Por ello, se procederá a exponer algunos de los aspectos centrales de su filosofía, teniendo como hilo conductor la nada, con el fin de comprender la fundamentación de su propuesta. Para así, finalmente aclarar el rol excepcional que se le atribuye en este pensamiento a la nada, como concepto filosófico.

\section{LA MUERTE DE DIOS Y EL ORIGEN DEL MUNDO}

La propuesta filosófica de Mainländer pretende develar el misterio del origen del universo, ello implica no sólo concebir y explicar el surgimiento de nuestro mundo, sino que de la existencia misma como tal. Aclarar el origen 
del todo conlleva también dar una respuesta al sentido de cada ser, es decir, aclarar su propósito. Esta es, sin lugar a dudas, una pretensión de grandes proporciones y de suma importancia para la filosofía: dar la respuesta al sentido de la existencia. Responder al por qué, para qué, y hacia donde, del ser.

Para comenzar el análisis del enigma sobre el origen, Mainländer asegura las bases de su investigación en un prolijo examen de nuestra capacidad cognoscitiva. En la primera sección de su obra magna encontraremos una Analítica de la facultad cognoscitiva donde se esclarecen y fundamentan los pilares fundamentales del análisis posterior. Cimientos tales como la ley de causalidad, el espacio, la materia, la síntesis, el tiempo, la sustancia, entre otros (Mainländer, 2014, p. 63).

Una vez teniendo en cuenta estos fundamentos, Mainländer se remonta hacia atrás en el tiempo, mediante un ejercicio de abstracción, yendo desde la multiplicidad en que actualmente nos encontramos hacia el pasado. Para que este retraimiento de la razón llegue a buen término, y no se quede infinitamente divagando de un objeto a otro en una serie causal infinita, Mainländer recurre a la construcción de series de desarrollo, una herramienta de la razón que tiene la ventaja de aferrarse a solo una cosa en sí hasta su origen:

La razón puede construir series de desarrollo [Entwicklungsreihen], que son algo completamente diferente de las series causales. Estas surgen con ayuda de la causalidad, mientras que aquellas surgen con ayuda del tiempo. Las series causales son la actividad encadenada, no de una, sino de muchas cosas; en cambio las series de desarrollo tienen que ver con el ser de una cosa en sí y sus modificaciones (Mainländer, 2014, p. 66).

De esta manera, al seguir el rastro de las diversas series de desarrollo, se desemboca en un dilema aparentemente irresoluble.

Por un lado, se es consciente de que, partiendo la reflexión dentro de los límites de este universo, resultaría imposible anular la pluralidad inmanente, y a lo sumo se podría llegar a la dualidad lógica. Mientras que, por otro lado, la razón exige la necesidad de una unidad a partir de la cual todo haya comenzado (Mainländer, 2014, p. 67).

La solución a este dilema estriba en concebir dicha unidad simple en un plano anterior al universo mismo. Este es el ámbito trascendente, que, al encontrarse en un pasado remoto, ya no existe más, y, por tanto, la unidad originaria tampoco: «Se trata de un dominio que ya pasó, que fue, que se hundió; y con él también pasó y se hundió la unidad simple» (Mainländer, 2014, p. 67). Por lo tanto, esta caracterización de la unidad simple, pierde todo su carácter chocante, al aclarar que es anterior a la multiplicidad del universo, es decir, que es una unidad precósmica [vorweltlichen].

Ante esta unidad primigenia las facultades humanas se petrifican, pues, 
si se es un recto investigador que se atiene a principios inmanentes -tal como Mainländer-, se reconocerá la incapacidad humana para profundizar en ella, sin caer en meros delirios metafísicos. En efecto, ante esta cabeza de medusa los sentidos resultan inútiles, el entendimiento no puede aplicar la ley de causalidad, ni las formas del espacio y la materia, asimismo, no hay nada que enlazar mediante la síntesis (Mainländer, 2014, p. 68). Es decir, todas aquellas cualidades de la razón que Mainländer reconoció y describió en su Analítica de la facultad cognoscitiva, son incapaces de indagar en el plano trascendente.

Solo es posible referirse a dicha unidad simple diciendo lo que no era, por contraste a aquellas cosas que conocemos, es decir determinarla negativamente como: «inactiva, inextensa, indiferenciada, indivisible (simple), inmóvil y atemporal (eterna)» (Mainländer, 2014, p. 68), el resto nos es irrepresentable.

Además, a dicha unidad simple, se le ha de reconocer una libertad absoluta. Esto porque al ser lo único que existía en aquel tiempo primigenio, no había nada exterior que le diera un motivo que la hiciera moverse con necesidad:

A la unidad simple debemos atribuirle la libertad, precisamente porque era una unidad simple. En ella queda suprimida la presión del motivo, uno de los factores de cualquier movimiento conocido por nosotros, pues ella no se había desunido aún, y permanecía enteramente sola y aislada (Mainländer, 2014, p. 136).

De igual modo, aquellos principios filosóficos de los que Mainländer se sirve para el conocimiento del mundo y sus relaciones (voluntad, espíritu y fuerza), son inaplicables a la unidad simple:

Tanto el principio fundamental, la voluntad, que en el dominio inmanente, resulta tan conocido e íntimo para nosotros, como el principio subordinado y secundario del espíritu y la fuerza, pierden, tan pronto como los traspasamos al dominio trascendente, toda significación para nosotros (Mainländer, 2014, p. 137).

Por ello, considerando todas estas salvedades, Mainländer se refiere a esta unidad precósmica simple con el nombre eminentemente trascendente e irrepresentable para la finitud humana:

Ahora bien, tenemos derecho a este ser el nombre con el que siempre se ha designado aquello que jamás ha alcanzado ninguna fuerza representativa, ni el más audaz vuelo de la fantasía, ni ningún pensamiento, por profundo y abstracto que haya podido ser; ni tampoco ningún sentimiento, aun siendo profundo y piadoso; ni, en fin, ningún éxtasis, por mucho que haya logrado apartarse de las cosas terrenales: el nombre de Dios (2014, p. 137).

Ahora bien, la concepción que desde esta filosofía se tiene de Dios, dista 
bastante de las tradicionales, ya sea desde la teología o desde el pensamiento filosófico anterior. Lejos de ser una divinidad que crea el universo como un acto de bondad, o para regir e interferir en el mundo y los asuntos humanos, el Dios mainländeriano es un Dios muerto, que, en el origen de todo, intentó precipitarse hacia la nada, fragmentándose y disolviéndose en el universo: «Dios ha muerto y su muerte fue la vida del mundo» (Mainländer, 2014, p. 137).

Ciertamente, esta novedosa cosmovisión le otorgó a la frase «Dios ha muerto» una nueva significación que, en el ámbito filosófico no se había dado.

Si bien la sentencia podría ser rastreada en otros momentos de la historia de la filosofía -unos años atrás hasta Hegel, y unos años después hasta Nietzsche-, no encontraremos en sus filosofías el mismo sentido inédito que tuvo para Mainländer.

En el sistema hegeliano la expresión adquiere una significación sumamente diferente. Para Hegel, la muerte de Dios no conlleva el génesis del universo mismo, sino que él habla, más bien, del «doloroso sentimiento de la conciencia desdichada de que es Dios mismo quien ha muerto» (Hegel, 2010, p. 891). Esto quiere decir, en su prosecución, que es un mero momento en la vida del espíritu, y que, por tanto, ha de quedar superado en el curso del devenir (Volpi, 2005, p. 31). De esta forma -para Hegel- la muerte de Dios no tiene un carácter fatalmente originario e imborrable, como si lo tiene en la filosofía de Mainländer.

En efecto, si nos mantenemos en el sistema hegeliano, se ha de considerar que aquella muerte de Dios, en el fondo alude al enaltecimiento de la razón moderna, y que, como tal, es una figura histórica que ha de ser acogida y superada, pues está llamada a desaparecer eventualmente (Restrepo, 2010, pp. 448-449).

Asimismo, si avanzamos unos años más en la historia de la filosofía, posterior a Mainländer, encontraremos que Nietzsche también se sirvió de esta polémica expresión, aunque en un sentido que tampoco es el que Mainländer le otorgó.

Seis años después de que la Filosofía de la redención ya circulara por Alemania, Nietzsche adoptó la frase en La gaya ciencia. Grabándola para la posteridad en el famoso aforismo 125, donde se lee: «¿Adónde ha ido Dios? [...] ¡yo os lo diré! ¡Nosotros lo hemos matado, vosotros y yo! ¡Todos nosotros somos sus asesinos!» (Nietzsche, 2014, p. 802). Esta proclamación tiene un carácter metafórico, que no alude a la no existencia de Dios, sino que más bien, a la constatación de su muerte, en cuanto cúspide de las ideas trascendentes que fundamentaban una moral decadente. En este sentido alegórico, se constata la muerte de Dios como un diagnóstico de la situación 
cultural de su época, entendiendo por Dios un símbolo de las principales ideas metafísicas en las que se creía y que Nietzsche criticaba.

Sin embargo, en la Filosofía de la redención, encontraremos esta sentencia en un sentido literal: «Esta unidad simple ha existido, pero ya no existe. Se ha hecho añicos, transformando su esencia completa y enteramente en el mundo de la pluralidad» (Mainländer, 2014, 137). Solo que, quien mató a Dios no fueron los hombres, sino él mismo, quien habría cometido lo que alegóricamente podemos representar como un suicidio divino, en un afán de transitar hacia la nada (Sevilla Godínez, 2014, p. 55). Dios no habría sido asesinado alegóricamente por los humanos en la modernidad, sino que, se habría suicidado en el origen. De esta manera, habría dejado de ser una unidad, y habría comenzado a ser la pluralidad, mediante su fragmentación, Dios muere y nace el universo, en un sentido literal y temporal.

La muerte de Dios y el origen del mundo son uno y lo mismo, el suicidio divino habría puesto en marcha todo lo que de ello devino, dando paso al surgimiento del mundo. En palabras de Mainländer: «El primer movimiento y el surgimiento del mundo son una y la misma cosa. La transformación de la unidad simple en el mundo de la pluralidad» (2014, p. 125). Ciertamente, en este tránsito, se marcó un curso universal, al que nada puede escapar: una tendencia profunda hacia la nada. Dios no se fragmentó en la multiplicidad para permanecer eternamente dividido, no fue un sacrificio en vano ni carente de meta, sino que lo hizo para entrar en un proceso de descomposición que acabaría ulteriormente disolviéndose en la nada.

Ahora bien, ¿por qué Dios elige la nada antes que el ser?, más aún, ¿por qué el ser supremo, una vez decido por adentrarse en la nada, no lo consigue de manera inmediata y, en lugar de ello, surge el universo?

Ante la primera pregunta Mainländer dirá que, en la total y absoluta libertad en que reposaba la unidad simple, solo le quedaba una única elección posible, ser o no ser: «la libertad de Dios (el liberum arbitrium indifferentiae) solo pudo hacerse valer en una única elección, a saber: o permanecer como era, o no ser» (2014, p. 336). Consecuentemente, si Dios hubiera querido libremente ser de cualquier otro modo, lo hubiera hecho sin problemas, de inmediato, pues gozaba de omnipotencia. Sin embargo, no fue el caso que haya elegido ser de un modo menos perfecto que tal como ya era: «El no ser ha debido, desde luego, ser preferido al supra-ser, pues, si no, Dios, con su perfecta sabiduría, no lo habría elegido» (2014, p. 339). En este punto se ve con claridad el fuerte pesimismo de este autor, para el cual la existencia es dolor, y el no ser es preferible al ser, lo cual también habría sido comprendido por Dios, y por ello anhelaba la nada absoluta (Beiser, 2016, p. 217).

Ante la segunda pregunta, Mainländer es aún más contundente, pues dirá que Dios -el ser supremo- era omnipotente ante el ser, pero impotente frente 
al no ser. Es decir, el único caso que restringiría la omnipotencia de Dios, sería la nada. Su omnipotencia no podía acabar con ella misma, es decir, el límite del poderío divino sería la nada:

[S]u poder no podía aniquilarse por sí mismo, ni la unidad simple podía cesar de existir por sí misma. [...]. Es este el único caso en el que la omnipotencia de Dios está restringida, precisamente por sí mismo; se ve que no era una omnipotencia contra sí mismo (2014, p. 338).

Pues, si Dios negara de modo inmediato su propio supra-ser, su omnipotencia no podría ya ejercerse de ningún modo. Consecuentemente, el ser absoluto, estaría en una situación deficiente ante la nada, debido a que esta última queda fuera de los límites del ser, incluso de los límites divinos del supra-ser mainländeriano.

En base a esto, podemos repensar lo que años más tarde dirá Heidegger, en un sentido diferente, aunque en esta misma línea sobre la nada y Dios: «Pero, si Dios es Dios, nada puede salvar de la nada, puesto que lo 'absoluto' excluye de sí toda nihilidad» (1996, p. 54). La alusión de Heidegger está en relación con el hecho de que, si nos figuramos un Dios que crea desde la nada -tal como el del cristianismo-, este tiene que habérselas con ella, lo cual conlleva serias dificultades y choques entre los conceptos de absoluto y nada. Sin embargo, estas oposiciones y problemas no se presentarían en la propuesta mainländeriana. Desde la Filosofia de la redención, se evitan las contradicciones y problemas en el encuentro entre la nada y lo absoluto, es decir, Dios.

Para Mainländer, en efecto Dios en cuanto Dios nada puede contra la nada, pues su omnipotencia se agota en el ámbito del ser. Algo en el supra-ser de la unidad precósmica le impedía adentrarse en la nada absoluta de un modo instantáneo: «su esencia fue el obstáculo para ingresar inmediatamente en el no ser» (2014, p. 340).

A Dios en cuanto Dios (supra-ser) le estaba vedada la posibilidad de la nada, pero en cuanto dejara de ser tal como era - una unidad simple, en cuanto dejara de ser Dios-, su esencia se iría paulatinamente debilitando hasta poder lograr el fin por el cual se había resuelto, la nada absoluta:

Una vez decidida por la existencia, vale decir, resuelta a sacrificar el superser por el no ser, la essentia presente tuvo que llevar a cabo la resolución y por eso, después de todo, se manifestó el mundo, pues en este proceso se trata únicamente de desembarazarse de la essentia, quebrar, debilitar y, por fin, exterminar el obstáculo (Mainländer, 2011, p. 121). 
En este sentido, según Mainländer, la unidad simple no crearía el universo desde la nada. Al contrario, Dios se habría fragmentado, muriendo en dicho acto suicida, con el fin de deshacerse del obstáculo que le impedía su objetivo. Deviniendo así en el universo, con el propósito de adentrarse en la tan anhelada nada: «A la unidad simple se le denegaba el logro inmediato de la meta, pero no conseguirla en general. Era necesario un proceso (un curso de desarrollo, un debilitamiento paulatino), y todo el curso de este proceso residía virtualiter en la fragmentación» (2014, pp. 347-348).

Por consiguiente, el cosmos no es una creación ni una emanación divina, sino que, son los restos de un Dios despedazado. El mundo es - para Mainländerdescomposición, un cadáver divino (Marcuse, 1956, p. 23). El universo en su totalidad, en toda su vasta finitud, no es más que los restos fragmentados de un Dios muerto, en un proceso de descomposición y debilitamiento, que tarde o temprano acabará, extinguiéndose hasta alcanzar la nada.

De este modo, Dios (el supra-ser) necesitaba del universo (el ser) para alcanzar su objetivo: la nada (el no ser). Meta que le era imposible alcanzar de modo instantáneo. El mundo, y el universo en su totalidad, son el punto de tránsito entre el supra-ser divino y la nada absoluta:

El mundo es el medio para alcanzar el fin del no ser, y el mundo es, por cierto, el único medio posible para alcanzar dicho fin. Dios conoció que solamente a través del devenir de un mundo real de la pluralidad; sólo a través del ámbito inmanente, es decir, del mundo, podría ingresar desde el supra ser en el no ser (Mainländer, 2014, p. 338).

Así, Dios devino mundo con su muerte, marcando el destino del universo, consagrándolo a la aniquilación, esto es, a alcanzar la meta que trazó con su suicidio: «ingresar en la nada absoluta, en el nihil negativum, es decir, aniquilarse por completo, cesar de existir» (Mainländer, 2014, pp. 336-337).

Ahora bien, para asegurarse de que dicha meta sería alcanzada, la unidad simple divina habría inoculado una tendencia en lo más profundo de toda la multiplicidad en que derivó su cadáver. Dios habría dejado una huella imborrable en cada individuo y cada cosa que de él devino: la voluntad de morir.

\section{VOLUNTAD DE VIVIR Y VOLUNTAD DE MORIR}

A diferencia de Schopenhauer, Mainländer no concibe una única voluntad cósmica eterna que impere de modo indistinto sobre todos los individuos. Pues pensar en una entidad trascendente -actualmente existente y activa- que mueva los hilos de todo cuanto hay, le parece a Mainländer algo inconcebible, un despropósito y una fantasmagoría. 
Para este autor, en cambio, dicha unidad existió en un tiempo pretérito, fue Dios en el inicio de los tiempos, en un plano trascendente que está solo en el pasado. Y por ello mismo, ya no existe más, puesto que se ha fragmentado en la multiplicidad del universo. Mainländer admite que existió efectivamente dicha unidad, pero Dios -la unidad precósmica- está muerto, ya que prefirió la no existencia por sobre la existencia (Dahlkvist, 2007, p. 79). Consecuentemente, no hay una única voluntad tras el cosmos, sino que hay muchas voluntades, tantas como individuos ${ }^{1}$ hay en él. Sin embargo, cada una de dichas voluntades individuales, tienen un único afán en común, el que se busca cumplir de diversas formas.

Habiendo descartado la posibilidad de una única voluntad cósmica universal schopenhaueriana, Mainländer plantea que tras cada ser de la multiplicidad hay una profunda e individual voluntad que no anhela la vida, sino que busca la muerte para consumar la redención (Beiser, 2016, p. 212).

En lo más profundo de cada individuo, se encuentra el anhelo de no ser. Esto porque el único deseo que Mainländer identifica en la unidad simple es, precisamente, el de desaparecer en la nada. De un modo similar, este anhelo seguiría estando en sus restos finitos: «El universo es efecto de deseo semejante. Que cada individuo se encuentre en el tiempo, que sea finito y mortal, significa que todo desea el no ser» (Espinoso Proa, 2019, p. 147). Consecuentemente, el rol que tienen los seres humanos en el devenir del cosmos, no es otro que concretar dicho anhelo originario.

No ha de confundirse, según Mainländer, no existe una única voluntad que opere sobre, tras o en el mundo, la cual se manifiesta en distintos seres particulares, sino que solo se reconoce una unidad antes del universo. Considerando esto, lo único que verdadera y actualmente existe son los múltiples individuos, y en cada uno de ellos una voluntad individual, con un único afán: la muerte, es decir, alcanzar el no ser.

La voluntad individual de morir es aquella huella que Dios impregnó en todo lo que de él devino con su muerte: «Cada voluntad individual sigue el impulso que surge del interior, es decir, persigue la estela que dejó la unidad precósmica» (Baquedano Jer, 2011, p. 30). Al morir Dios, y convertirse en el cosmos, legó en los individuos una aspiración por concretar su meta: la completa aniquilación. Dicha voluntad suele manifestarse de modo aparente

1 Ha de comprenderse el término individuo en un sentido amplio, ya que Mainländer considera que cada cosa, independiente de si es orgánica o inorgánica, es una idea con una voluntad individual. De este modo, tanto el ser humano, animales, plantas y hasta elementos químicos, poseen vida, $\mathrm{y}$, consecuentemente voluntad. Respecto a la atribución de vida al reino inorgánico véase el $\S 19$ de la Física de Mainländer (2014, pp. 105-106), así como también el §25 de la misma sección (2014, pp. 111-112). Respecto al reino vegetal véase el §14 de la misma sección (2014, p. 104). Respecto al reino animal véase el $\S 15$ de la misma sección (2014, pp. 100-102). 
como voluntad de vivir, en cuanto la vida es el mejor medio para la muerte, pero en su fondo originario, es pura voluntad de morir, es decir, anhelo de no ser y de la redención que ello conlleva. Redención que se cumplirá con necesidad, mediante el constante desgaste y debilitamiento de las voluntades de vivir, en su ferviente afirmación.

De este modo, Mainländer reconoce que, ante una primera mirada, puede constatarse que cada ser anhela preservar su existencia, quiere la vida: "Queremos estar ahí, y para siempre; porque queremos la existencia, existimos, y como queremos la existencia permanecemos en ella. La voluntad de vivir es el núcleo más íntimo de nuestro ser» (2014, p. 81). Tal conclusión provisional es propia de un examen que se atiene a un punto de vista inferior en la naturaleza. Sin embargo, tras ahondar con mayor profundidad, se puede reconocer que hay un anhelo más profundo tras cada ser, ante el cual la voluntad de vivir está subordinada: «Pero, en el fondo, el filósofo inmanente ve en todo el universo solo el más profundo anhelo de absoluta aniquilación» (2014, p. 347). Así, todo en el mundo sería pura voluntad individual de morir, un afán de ir hacia la nada. Mientras que, el anhelo por la vida es solo una manifestación de dicha voluntad de muerte más profunda. Pues sólo mediante la existencia de múltiples seres finitos que se empeñen en afirmar la vida debilitándose en dicho proceso-, puede alcanzarse la meta final: la redención de la existencia al adentrarse en el nihil negativum.

La vida es el mejor medio para la muerte, a través de un extenso proceso de fragmentación en diversos individuos en constante lucha, las fuerzas en ellos se van debilitando cada vez más. En dicho proceso existe una tendencia que guía igualmente a cada cual: «en este tender, dichos seres se obstaculizan mutuamente, luchan unos con otros, $\mathrm{y}$, de este modo, debilitan su fuerza» (Mainländer, 2014, p. 340). Por consiguiente, la voluntad de morir, que opera en distintos grados en cada uno de estos individuos sería «la conciencia de la vida como medio para alcanzar la liberación a través de la muerte» (Baquedano Jer, 2007, p. 122).

La voluntad de morir sería el núcleo más interno de cada individuo, independiente de su complejidad o simpleza. De este modo, todo el reino inorgánico tiene sólo un afán, la total aniquilación, por lo que, en todos los elementos y compuestos químicos -que son la base de todos los organismos más complejos- reposa un incesante anhelo de muerte: «En todo el reino inorgánico no es querida la vida, sino la aniquilación; lo que se quiere es la muerte. [...]. La vida no es querida, sino que sólo es manifestación de la voluntad de morir» (Mainländer, 2014, p. 343). De manera similar, existiría el mismo querer ciego en el reino orgánico, pero de modo cada vez más oculto según la complejidad del organismo. Por ello, Mainländer dirá que en las plantas se da la voluntad de morir junto a la voluntad de vivir (2014, p. 
344), mientras que en los animales esta última se da antes (2014, p. 345), es decir, el medio se antepone al fin, y finalmente, en los seres humanos, al ser organismos más complejos, la voluntad de vivir eclipsa completamente en lo más profundo a la voluntad de morir (2014, p. 346). No obstante, mediante la razón, es posible revertir dicha situación y tomar consciencia de ello.

Desocultando la voluntad de morir, eclipsada por la voluntad de vivir, el ser humano es capaz de comprender que dicho afán no es un mero capricho particular, que solo le concierna a sí mismo, o a su especie, sino que es el núcleo más íntimo de todo en el universo. En tanto que se entiende ahora que, es un anhelo de redención, el cual se remonta hasta el origen del universo, es decir, hasta la muerte de Dios. Esta toma de consciencia mediante el desocultamiento de la voluntad permite tener una nueva perspectiva ante el curso del universo, como el despliegue del único anhelo divino y originario:

Así, lo que se oculta tras la aparente voluntad de vivir de los individuos no es sino la misma voluntad de muerte que condujo a Dios a suicidarse. Los diferentes meandros de la voluntad de vivir en la historia (las distintas civilizaciones, por ejemplo) no son en realidad otra cosa que tentativas - cada vez más refinadas-de la voluntad de morir de Dios, en su empeño por alcanzar cuanto antes el no ser (Fronteala Asensio, 2019, p. 302).

Cada individualidad -sea un elemento químico, planta, animal o ser humano-, independiente de su complejidad es profunda y fundamentalmente voluntad de morir. En tanto que cada cosa en el universo es un fragmento de Dios, que se limita seguir el movimiento que le fue dado en su origen y a dar cumplimiento al destino entrópico universal.

De modo análogo, el curso de la humanidad mediante el proceso de civilización, no es otra cosa que adentrarse más aceleradamente en el rumbo hacia la aniquilación. De esto no hay excepción, el declive de las civilizaciones estriba en su acelerado movimiento que acerca cada vez más a los pueblos civilizados hacia su extinción:

Podemos reconocer con claridad la importante verdad de que la civilización mata. Cualquier pueblo que ingresa en la civilización, es decir, pasa a poseer un movimiento más rápido, cae y se estrella en las profundidades. Ninguno puede mantenerse en su fuerza humana, sino que debe disfrutar de la vida, volverse decrépito y degenerar (Mainländer, 2014, p. 278).

El proceso de civilización confluirá finalmente en la aparición del Estado ideal, una forma histórica que abarque a toda la humanidad, en la que se 
suprimirán las miserias y desigualdades. En tal escenario, la vida podrá ser juzgada rectamente, y los ciudadanos actuarán acorde a ello:

Su espíritu juzga ahora correctamente la vida, y su voluntad se enardece con este juicio. Ahora lo único que aún llena el corazón es un único anhelo: ser tachado para siempre del gran libro de la vida. Y la voluntad alcanza su meta: la muerte absoluta (Mainländer, 2014, p. 325).

De este modo la humanidad hará el «gran sacrificio»: adentrarse totalmente en la nada. Pues, desde una perspectiva más elevada, el conjunto de la humanidad no es más que un fragmento entre otros de lo que pretéritamente fue la unidad precósmica, que se encuentra debilitándose en el universo en un proceso de autocadaverización de Dios (Volpi, 2005, p. 48). Este es un proceso del cual no podemos escapar ni contrarrestarlo, ya que sigue un curso fatalmente necesario hacia la nada.

Según Mainländer, la humanidad al estar dentro de este inmenso proceso, no tiene otra opción que dirigirse de modo retardado hacia la nada, cada individuo $-\mathrm{y}$, por tanto, la totalidad- sería arrastrado hacia la muerte, con la fuerza de una unidad simple.

En este trayecto, los individuos que se aferran a la vida no hacen más que debilitarse lentamente, su movimiento sería tal como un espiral descendente, que va desde la vida hacia la muerte absoluta $(2014$, p. 328). El rol que le queda al individuo es únicamente comprender este tránsito inevitable, y adecuarse a él, pues nada puede hacerse para detenerlo, ni siquiera si toda la humanidad en su conjunto lo intentara de modo simultáneo. Pues cada cosa quiere la muerte en su más recóndito subterfugio, y es una condición que no se puede modificar, inherente al ser mismo de cada individuo. Según esta cosmovisión, somos fragmentos de un Dios destrozado, que se van deshaciendo más y más a medida del tiempo, hasta acabar en la nada (Vega Delgado, 1991, p. 455).

Quien tome consciencia de la situación del ser humano en el curso del universo, puede hacer algo más que simplemente aterrorizarse por el inexorable destino del ser. Aquel que asuma este tránsito hacia el no ser, no puede hacer nada para evitarlo, pero si para acelerarlo, esto mediante la negación de la voluntad de vivir (afirmación de la voluntad de morir).

Aquellos que sean conscientes de dicho curso inevitable, podrán con algunas acciones acelerar la entrada en el no ser, ajustándose al movimiento que va desde el ser hacia la nada. La afirmación de la voluntad de morir puede efectuarse mediante una vía ascética ante la vida, la cual puede adoptar, en ciertas circunstancias actitudes como la virginidad y, en ciertos casos más extremos el suicidio. Ambas son actitudes de auto supresión que, según Mainländer, no van contra la naturaleza, sino que ocurren con estricta 
necesidad y se ajustan de mejor modo al curso del cosmos, ya que debilita más eficazmente la suma de fuerzas (2014, p. 351).

No obstante, la vía de la afirmación ferviente de la voluntad de vivir, consigue a fin de cuentas el mismo fin. Mediante la lucha y debilitamiento constante de los individuos, cada vez se está más cerca de su total aniquilación. Por tanto, la voluntad de morir opera fuertemente en ambas vías: tanto si se es consciente del curso del cosmos y se opta por afirmar la muerte, o bien, si se está cegado por el derroche de fuerzas vitales, y se vive y afirma la vida intensamente. De ambas formas, todo avanza irrevocablemente hacia un mismo fin: la nada absoluta.

Desde la antípoda de la afirmación de la voluntad de morir, la virginidad se presenta como la renuncia segura a la voluntad de vivir (Dahlkvist, 2007, 81). Consecuentemente, si se quiere alcanzar la redención anticipadamente, y de modo seguro, la voluntad ha de experimentar una transformación que la haga amar la muerte: «Pero ahora la voluntad no solo debe despreciar la muerte, sino que ha de amarla; de manera que la castidad es amor a la muerte» (Mainländer, 2014, p. 237).

Asimismo, el suicidio también se podría caracterizar como una acción que se ajusta al curso del cosmos. Si se da en consonancia con la virginidad, y no se han dejado descendientes al momento de morir, entonces, el suicida habrá sido tachado para siempre de la existencia y se habrá redimido, adentrándose en la nada absoluta. Ante todo, se ha de tener en cuenta que, Mainländer no lo recomienda como un paso necesario de la vía ascética, por tanto, no sería adecuado decir que haga un llamado a ello. El suicidio no es una consecuencia necesaria del reconocimiento del curso del universo. Pues, se obtendría el mismo resultado al final de la vida por una muerte natural, siempre y cuando no se haya dejado descendientes sobre el mundo.

La filosofía de Mainländer no hace un llamado a un suicidio colectivo ni mucho menos, pero sí depura este acto de todas las condenas morales que suelen recaer sobre él: «La filosofía inmanente no puede permitirse condenar, ni puede hacerlo. No exhorta al suicidio; pero en aras de la verdad, debió destruir los poderosos motivos que se le oponen» (2014, p. 360), reconociendo que esta acción no ha de ser reprobada por sí misma, y que incluso ayuda a acelerar el curso del cosmos hacia la nada (del mismo modo que lo haría, en su medida, la posición opuesta, afirmando ciegamente la vida).

Si bien en Mainländer no encontramos dicha recomendación o llamado al suicidio, tampoco encontraremos una postura absolutamente reprobatoria ante este acto. Aquellos que salgan de la vida por mano propia, bajo estas circunstancias, no han de esperar un más allá trascendente que los castigue o premie por su decisión, sino que, solo encontrarán la nada, la cual consciente o inconscientemente, ha sido siempre anhelada en lo más profundo por cada 
uno: «Hermanos míos, salid sin temblar de esta vida, si carga con tanto peso sobre vosotros: no encontraréis ni un reino celestial ni un infierno en la tumba» (Mainländer, 2011, p. 130).

Estas actitudes prácticas de auto supresión -virginidad y suicidio-, en la medida en que son producto de un saber sobre el origen y destino del cosmos, son las herramientas de las que dispone el ser humano para obtener la redención de un modo anticipado, es decir, de alcanzar la nada absoluta antes que otros: «La castidad y el suicidio, cuando son realizados de manera consciente a través del conocimiento de la esencia del mundo, son los únicos medios para llevar a cabo aquella finalidad: la nada absoluta, objetivo para el cual el mundo apunta inevitablemente» (Caldeira Ramos, 2007, p. 42).

Ciertamente, en la medida en que todo tiende inevitablemente a la nada, todos serán redimidos ulteriormente, pero hasta entonces padecerán los sufrimientos inherentes a la existencia. Sin embargo, aquellos que opten por estas acciones, y se sirvan de los medios para obtener la redención, tendrán la ventaja de salir antes de la existencia, y poner término a los sufrimientos de la vida.

Cada individuo, al adentrarse en la nada absoluta, no solo se redime a sí mismo, sino que también contribuye a que la totalidad del universo esté un paso más cerca de la absoluta redención: "allí donde un ser se redime, Dios está más cerca de alcanzar la finalidad de su obra, el no ser» (González Serrano, 2019, p. 44).

\section{EL SENTIDO TELEOLÓGICO REGULATIVO DE LA NADA}

En el inicio del todo, la unidad precósmica simple que fue Dios anhelaba el no ser -la nada absoluta-, en base a dicho anhelo fue «creado» el universo con una huella que lo impulsa a alcanzar el único fin trazado en el origen. En este sentido, para Mainländer, la nada tiene una importancia fundamental dentro de su sistema filosófico, no solo tiene preeminencia metafísica sobre el ser (Caldeira Ramos, 2007, p. 40) lo que hace que sea preferible no ser antes que ser. Sino que, además, tiene un sentido teleológico, es decir, es la causa final del universo: «La única causa final que puede admitir el filósofo inmanente es la nada; sin embargo, determina expresamente que esta única causa final sólo puede ser establecida y utilizada de manera regulativa» (Mainländer, 2011, p. 65).

Son diversos los pasajes en que este filósofo se refiere a la finalidad o meta del cosmos, sin embargo, no por ello renuncia a sus principios inmanentes. Consecuentemente, cada vez que se refiere al fin al cual todo apunta y se dirige, lo hace con esta salvedad: refiriéndose a ella de modo regulativo. De esta manera se blinda de posibles críticas, del mismo modo en que se refirió de modo regulativo a la eventual existencia pasada de la unidad precósmica 
que fue Dios.

La nada tiene para Mainländer, por tanto, un sentido teleológico regulativo, en el significado que Kant le daba a los principios regulativos. Es decir, como una capacidad de la razón de indicar el camino que han de tener las cosas en aquellos asuntos donde la experiencia ya no pueda dar cuenta:

Es ciertamente un principio regulativo de la razón, legítimo y excelente; [principio] que como tal, empero, va mucho más allá de donde pudieran seguirle la experiencia o la observación, pero sin determinar nada, sino sólo para indicarle a ella el camino de la unidad sistemática (Kant, 2007, p. 706).

Mainländer reconoce la utilidad y provecho que representa la teleología, pero a la vez procura no abusar de ella, por lo que se asegura de emplearla con la mayor cautela, siempre en un sentido regulativo, tal como lo toma de Kant (Mainländer, 1996, p. 484).

En este sentido, Mainländer se sirve de la teleología para ir más allá de la experiencia y poder determinar el curso del universo: «La teleología es un principio meramente regulativo para el enjuiciamiento del curso del mundo» (2014, p. 348). De la misma forma en que la teleología sirve para remontarnos y enjuiciar el origen del universo, nos sirve para determinar el fin hacia el cual se dirige. Dicho enjuiciamiento no tiene la pretensión de una certeza apodíctica, sino que se limita a expresar cómo se presentan las cosas para el ser humano: «Por consiguiente, todo tiene una meta en el mundo, o mejor: la Naturaleza se presenta para el espíritu humano como si se moviese hacia una meta única» $(2014$, p. 348).

De este modo, Mainländer establece junto con la finalidad del curso del mundo, el sentido de la vida, el cual no es otra cosa que la muerte absoluta. El ser humano puede alcanzarla si se cumplen ciertas condiciones ${ }^{2}$, la muerte representa para este autor el paso final hacia la nada absoluta, el fin de toda experiencia humana, en otras palabras, el sentido de la vida (Beiser, 2016, p. 212).

Este sentido de la vida para el ser humano es el mismo que el del universo: la nada absoluta, la muerte. Esto porque Dios así lo quiso, se ponderó en el origen que el no ser es preferible al ser, y se inició todo un proceso cósmico que tiene un único objetivo, el nihil negativum. Por ello, todo cuanto hay en

2 Si no se han dejado hijos sobre la tierra, es decir, siempre y cuando el individuo no perviva más en el plano inmanente. Respecto a la procreación humana entendida como rejuvenecimiento de los progenitores véase el §26 de la Física de Mainländer (2014, pp. 112-115). En lo concerniente a la virginidad como mejor forma de evitar dicho rejuvenecimiento y alcanzar la redención véase el §26 de la Ética de Mainländer (2014, pp. 236-238) y §28 (2014, pp. 239-241). Asimismo, véase también el $§ 17$ y $§ 18$ de la Metafísica del mismo autor (2014, pp. 350-353). 
el universo quiere su muerte, de un modo inconsciente mediante la profunda voluntad de morir. Porque Dios en su primer y único acto -su suicidio divino-, trazó el destino del mundo: «Esta unidad quiso el no ser y por eso conspira todo en el universo y en el individuo para el no ser» (Mainländer, 2011, p. 66).

Ateniéndose a este sentido teleológico regulativo de la nada, habría que añadir que, todo cuanto sucedió en el curso del mundo hasta ahora, lo que actualmente está ocurriendo, así como lo que pasará en un futuro: «todo sucedió y sucede, hablando de forma figurada, por mor de una única meta, el no ser» (Mainländer, 2014, p. 353). Esa manera figurada de expresarse corresponde al cauteloso matiz mainländeriano que reviste a la teleología con un carácter regulativo.

En este mismo sentido Mainländer recurre de nuevo a las series de desarrollo que al comienzo se usaron para remontarse al origen (2014, p. 66), y se puede fijar que al proyectarlas hacia el futuro estas también tienen un fin, no son infinitas, pues culminan en la meta a la que cada ser se dirige:

Todo el universo se mueve desde el ser al no ser, debilitándose continuamente su fuerza, y las series de procesos a las que debimos ya otorgar un comienzo en la Analítica, han de tener también un fin; no son infinitas, sino que desembocan en la pura y absoluta nada, en el nihil negativum (2014, p. 353).

Teleológicamente desembocan en su extinción, es decir, el desarrollo de cada cosa en sí, cada individualidad, ulteriormente se desintegrará en la nada absoluta. Entonces, y solo entonces, se habrá consumado el acto de fragmentación divina, cuando el último individuo se redima y se alcance el objetivo: «Luego, Dios, desde el supra-ser y a través del devenir, ingresa en el no ser, encontrando, a través del proceso universal, aquello que impedido por su esencia no podía alcanzar inmediatamente: el no ser» (Mainländer, 2014, p. 356).

Ante esto, el filósofo del Dios muerto reconoce que puede haber ciertas objeciones, que traten de postular algo diferente como meta final, tras la vida y el curso del universo. Por ejemplo, que al final, la meta que se alcanzaría sería un paraíso (Mainländer, 2011, p. 75).

Sin embargo, esta es una objeción que sería insostenible a los ojos de Mainländer. Ya que, si Dios hubiera querido ser un conjunto de seres que gozaran de bienes en un paraíso, lo hubiera conseguido de inmediato gracias a su omnipotencia, por lo que no necesitaría de ningún proceso de devenir para ello. Razón por la cual, el único objetivo posible para este proceso es devenir hacia lo único que no podía obtener inmediatamente la omnipotencia divina: el no ser.

Descomponer hasta la nada, lo que alguna vez fue Dios, tal es la razón de ser de cada ente en el universo. En efecto, no puede haber otra cosa más allá 
de la muerte ni al final del proceso cósmico en que nos encontramos, salvo el nihil negativum: «Más allá de este mundo no hay, ni un lugar de paz, ni de tormento, sino solamente la nada. Quien ingrese en ella, no tiene ni reposo, ni movimiento; carece de estado alguno, [...], la voluntad está completamente aniquilada» (Mainländer, 2014, p. 361). La nada hacia la que todo confluye, se encuentra, por tanto, en un nivel metafísico, más allá de nuestra capacidad de concebirlo: «El universo es el punto de tránsito, pero no para un estado nuevo, sino para la aniquilación, la cual, desde luego, se encuentra fuera del universo, es metafísica» (Mainländer, 2011, p. 76).

Esta aniquilación coincide con adentrarse en la nada, es la meta del tortuoso tránsito que es la existencia, desde esta postura pesimista. De este modo puede comprenderse como todo el género humano está consagrado por igual a la muerte absoluta, lo que lejos de ser algo malo, es el mejor de los escenarios posibles, dentro de los márgenes de este fuerte pesimismo. Puesto que la existencia es dolor, la muerte -en tanto constituye un adentrarse a la nada- representa el mejor modo de ponerle término a dicho sufrimiento, alcanzar la nada equivale a la redención (Mainländer, 2014, p. 347). Por ello cabe denominar a este sentido teleológico de la nada como una generosa escatología redentora (Beiser, 2016, p. 219), pues la redención no está reservada únicamente para unos cuantos elegidos, o para quienes arduamente hagan sacrificios y esfuerzos para conseguirla, sino que, así como la muerte le es común a todos, la nada redentora también llegará tarde o temprano a cada ser.

Sin embargo, Mainländer es consciente de que, muchos, que no estén aún maduros para la muerte, verán con temor a la nada absoluta que habrá como meta final. Y por ello es indulgente con aquellos que prefieran representarse de modo diferente y adornado aquello que aguarda al final del transcurso del universo, ya que «El hombre tiene una tendencia natural a personificar el destino, y a concebir místicamente la nada absoluta, que le mira fijamente a los ojos desde cada tumba» (2014, p. 367), sin embargo, el final será para todos el mismo. Ya sea que se figure un reino de los cielos junto a un Dios Padre personal, o bien se sea capaz de aceptar el sentido teleológico regulativo de la nada, y mirar fijamente a los ojos, con alegría a la nada absoluta para adentrarse en ella.

La propuesta filosófica de Mainländer es, ciertamente llamativa en el plano teórico, sin embargo, puede parecerle al lector de la Filosofía de la redención difícil de mantener y concretar en la práctica, ser consecuente con dichos postulados. No obstante, el autor de esta obra filosófica si ajustó tanto su vida como su muerte a sus reflexiones sobre el origen, curso y finalidad del mundo. Tal como lo propuso en sus escritos, sin dejar hijos sobre la tierra, y 
tras recibir la primera edición impresa de su obra capital, el joven Philipp Batz se suicidó la noche del 1 de abril de 1876 (Baquedano Jer, 2011, p. 17).

Un final que, años antes, en el terreno de la poesía había esbozado en su diario, mediante los siguientes versos:

\author{
Así pues, ¡que mi muerte tache del libro de la vida \\ el ser de mi alma para siempre! \\ Pues quien no rejuvenece el alma en los niños, \\ el ser entero del alma en la muerte pierde. \\ De esta alegre y señera certeza \\ llena está el alma abierta y benevolente; \\ y me es igual fundirme con la nada, \\ que con la divina paz desposarme. ${ }^{3}$ \\ (Mainländer, 2015, p. 179).
}

\title{
REFERENCIAS BIBLIOGRÁFICAS
}

BAQUEDANO JER, Sandra (2007). «¿Voluntad de vivir o voluntad de morir? el suicidio en Schopenhauer y Mainländer». Revista de Filosofía, 63, pp. 117126.

(2011). «Estudio preliminar, el pesimismo entrópico en las cosmologías filosóficas de la voluntad», en P. Mainländer, Filosofía de la redención (antología). Santiago: Fondo de Cultura Económica,, pp. 9-39.

BEISER, Frederick (2016). «Mainländer's Philosophy of Redemption», en F. Beiser, Weltschmerz: Pessimism in German Philosophy, 1860 - 1900, New York: Oxford University Press, pp. 201-228.

BURGOS CRUZ, Oscar, \& Godoy Patiño, Iliana (2015). «Sobre una posible influencia de Mainländer en Freud», Tlamati, 6, pp. 6-18.

CALDEIRA RAMOS, Flamarion (2007). «O pessimismo e a questão social em Philipp Mainländer». Cuadernos de Filosofia Alemã, 10, pp. 35-50.

DAHLKVIST, Tobias (2007). «Nietzsche and the Philosophy of Pessimism». Tesis. Uppsala Universitet.

ESPINOSO PROA, Sergio (2019). «Del deseo de no ser», en O. Burgos Cruz (coord.), Philipp Mainländer. Actualidad de su pensamiento, Guerrero: Universidad Autónoma de Guerrero, pp. 154-152.

FRONTEALA ASENSIO, Pablo (2019). «La libertad como identificación con la necesidad en Philipp Mainländer», en J. Chillón, P. Fronteala \& Á. Martínez (eds.), Hombre y logos: Antropología y comunicación, Madrid: Fragua, pp. 295-303.

3 Fragmento de la segunda parte del poema $\mathrm{n}^{\circ} 45$ (invierno), escrito en 1863 . El énfasis es mío. 
GONZÁLEZ Serrano, Carlos (2019). «Libertad fragmentada: voluntad de morir y muerte de Dios en Philipp Mainländer», en O. Burgos Cruz (coord.) Philipp Mainländer. Actualidad de su pensamiento, Guerrero: Universidad Autónoma de Guerrero, pp. 37-46.

HEGEL, Georg. Fenomenología del espíritu. Madrid: Abada, 2010.

HEIDEGGER, Martin. ¿Qué es metafisica?; Ser, verdad y fundamento: Ensayos. Buenos Aires: Siglo Veinte, 1996.

KANT, Immanuel. Crítica de la razón pura. Buenos Aires: Colihue, 2007.

MAINLÄNDER, Philipp (2015). Diario de un poeta. Madrid: Plaza y Valdés. (1996). Die Philosophie der Erlösung I. New York: Georg Olms Verlag. (2011). Filosofía de la redención (Antología). Santiago: Fondo de Cultura Económica.

(2020). Filosofía de la redención (y otros textos) Antología. Madrid: Alianza. (2014). Filosofía de la redención. Madrid: Xorki.

(2018). Rupertine del Fino. Madrid: Guillermo Escolar.

MARCUSE, Ludwing (1956). Pesimismo un estado de madurez. Buenos Aires: Leviatán.

NIETZSCHE, Friedrich (2014). «La gaya ciencia», en D. Sánchez Meca (ed.), Obras completas III, obras de madurez I. Madrid: Tecnos, pp. 704-905.

RESTREPO, Carlos (2010). «La frase de Hegel: Dios ha muerto». Escritos, 41, pp. 427-452.

SEVILLA GODÍNEZ, Héctor (2014). «El suicidio de Dios. La apología del exterminio humano en Philipp Mainlander», en H. Sevilla Godínez (coord.) Analogías alternantes de la nada. Madrid: Plaza y Valdés, pp. 51-76.

VEGA DELGADO, José (1991). La filosofía alemana, prolegómenos para una superación de la filosofía. Cuenca: Universidad de Cuenca.

VOLPI, Franco (2005). El nihilismo. Buenos Aires: Biblos.

PaOlo Gajardo JaÑa es licenciado en Filosofía y actualmente cursa un Magister en Filosofía en la Universidad de Chile.

Lineas de investigación:

Pesimismo, nihilismo, metafísica

Publicaciones recientes:

(2020). «Reseña de Extravíos», Revista Hybris 11(1), pp. 307-310.

(2018). «El lirismo como método de canalización agónica de la irracionalidad en el pensamiento de Cioran», Revista Mutatis Mutandis 10, pp. 75-92.

Correo electrónico: paolo.gajardo@ug.uchile.cl 
